\title{
Mechanisms for spin suppression and orbital enhancement in $M 1$ transitions: Critical assessment
}

\author{
L. Zamick and D. C. Zheng \\ Department of Physics and Astronomy, Rutgers University, Piscataway, New Jersey 08855 \\ E. Moya de Guerra \\ Instituto de Estructura de la Materia, Consejo Superior de Investigaciones Científicas, 28006 Madrid, Spain
}

(Received 7 February 1989)

\begin{abstract}
We discuss different nuclear structure properties that may lead to spin suppression in magnetic dipole excitations (as well as in Gamow-Teller transitions). We especially compare the triaxial and the axial asymptotic deformation limits and show that the summed $B(M 1)$ strength can be quite different in the two cases. A connection between triaxial deformation and spin suppression is established. It is known, however, that modern Hartree-Fock calculations yield axially symmetric solutions for several nuclei which were previously thought to be triaxial. We show that taking into account the effects of higher shells can lead to an enhanced orbital contribution to $M 1$ excitations without affecting ground-state magnetic moments.
\end{abstract}

\section{GENERAL CONSIDERATIONS, DEFINITIONS, AND SUM RULES}

For the purpose of our discussion in this and the next sections, it is useful to start with a few standard definitions. In terms of the magnetic dipole operator

$$
\boldsymbol{\mu}=\mu_{N} \sum_{i}\left(g_{l}^{i} \mathbf{1}_{i}+g_{s}^{i} \mathbf{s}_{i}\right)
$$

we write the transition strength from initial state $\left(I T T_{z}\right)$ to final state $\left(I^{\prime} T^{\prime} T_{z}^{\prime}\right)$ as

$B(M 1 ; i \rightarrow f)=\frac{1}{2 I+1} \frac{3}{4 \pi}\left|\left\langle I^{\prime} T^{\prime} T_{z}^{\prime}\|\mu\| I T T_{z}\right\rangle\right|^{2}$,

where $I, T$, and $T_{z}$ stand for total angular momentum, isospin, and its third component, respectively. The total $M 1$ strength from ground state $(i=$ g.s. ) is simply denoted by $B(M 1) \uparrow$ :

$$
\begin{aligned}
B(M 1) \uparrow & =\sum_{f} B(M 1 ; \text { g.s. } \rightarrow f) \\
& =\frac{3}{4 \pi} \sum_{n \neq \text { g.s. }} \mid\left.\langle n|\mu| \text { g.s. }\rangle\right|^{2},
\end{aligned}
$$

and the linear energy weighted sum rule (LEWSR) by

$$
\begin{aligned}
B_{1}(M 1) \uparrow: & \\
B_{1}(M 1) \uparrow & =\frac{3}{8 \pi}\langle\text { g.s. }|[\mu,[H, \mu]]| \text { g.s. }\rangle \\
& =\frac{3}{4 \pi} \sum_{n}\left(E_{n}-E_{\text {g.s. }}\right) \mid\left.\langle n|\mu| \text { g.s. }\rangle\right|^{2} .
\end{aligned}
$$

The quenching of spin and/or orbital strength in $M 1$ transitions can be easily related to symmetries of the nuclear Hamiltonian. In that respect, Eq. (3) is particularly useful to analyze the role of symmetries and the role of different nuclear interactions in $M 1$ excitations. It is also convenient (and a common practice) to decompose the magnetic dipole operator into an isoscalar and an isovector operator

$$
\boldsymbol{\mu}=\left(\boldsymbol{\mu}_{S}-\boldsymbol{\mu}_{V}\right) \mu_{N}
$$

with

$$
\begin{aligned}
& \mu_{S}=\frac{1}{2} \sum_{i} \mathbf{j}_{i}+\mu^{S} \sum_{i} \sigma_{i}, \\
& \mu_{V}=\sum_{i} 1_{i} t_{z}^{i}+2 \mu^{V} \sum_{i} \sigma_{i} t_{z}^{i},
\end{aligned}
$$

where we use the conventions

$$
\begin{aligned}
& t_{z}=+\frac{1}{2} \text { neutron, }-\frac{1}{2} \text { proton }, \\
& g_{l, s}=\left(g_{l, s}\right)_{\text {bare }}, \\
& \mu^{V}=\frac{1}{4}\left(g_{s}^{p}-g_{s}^{n}\right)=2.353,
\end{aligned}
$$

and $\mu^{S}$ is the Morpurgo factor,

$$
\mu^{S}=\frac{1}{4}\left(g_{s}^{p}+g_{s}^{n}-1\right)=0.19 \text {. }
$$

The first term of $\mu_{S}$ commutes with $H$ and does not induce transitions. The isoscalar spin term is hindered by the Morpurgo factor ${ }^{1}$ and in the $L S$ coupling limit is also diagonal. One then sees that $M 1$ excitations are essentially isovector. Because of the hindrance factor $1 /\left(2 \mu^{V}\right)$ in the orbital contribution relative to the spin one, most $M 1$ excitations are predominantly of spin isovector character. Hence, traditionally, it is the spin isovector term in Eq. (5),

$$
\mathbf{Y}_{z}=\sum_{i} \sigma_{i} t_{z}^{i}
$$

that has been extensively studied. Further interest in this operator stems from its close relation to the GamowTeller (GT) operators of $\beta$ decay,

$$
O^{\mathrm{GT}( \pm)}=\mathbf{Y}_{ \pm}=\sum_{i} \sigma_{i} t_{ \pm}^{i}
$$


which, together with the spin (s) and isospin ( T) operators, form the generators of the SU(4) Wigner symmetry group. ${ }^{2}$

However, in the last few years several $M 1$ excitations have been found ${ }^{3}$ that are predominantly orbital, and interest in the isovector orbital term has increased. The appearance of these excitations, together with the longstanding problem of the quenching of spin excitations and Gamow-Teller strengths, makes it interesting to analyze these operators on the same footing.

In the next section we shall discuss selection rules that emerge from (a) shell-model calculations with simplified two-body interactions and (b) calculations with Nilssontype one-body Hamiltonians. But, before doing so, it is useful to consider some general properties and their rela- tions with the sum rules in Eqs. (2) and (3). These sum rules have been extensively discussed in the literature for the isovector spin operator ${ }^{4}$ and to a much lesser extent for the isovector orbital operator. If we consider a nuclear Hamiltonian of the form

$$
H=\sum_{i}\left[\mathrm{p}_{i}^{2} / 2 m+V\left(r_{i}\right)\right]+\sum_{i} a_{i} \mathbf{1}_{i} \cdot \dot{\boldsymbol{\sigma}}_{i}+\sum_{i<j} V_{i j},
$$

with $V_{12}$ a two-body potential of the simplified form

$$
V_{12}=V\left(r_{12}\right)\left(W+B P_{12}^{\sigma}\right) \text {, }
$$

the LEWSR's for the spin $\left(S_{V}\right)$ and orbital $\left(O_{V}\right)$ isovector operators are

$$
\begin{aligned}
& B_{1}^{S_{V}}(M 1) \uparrow=-\frac{3}{4 \pi}\left(3 \mu_{N} \mu^{V}\right)^{2}\left\{\left\langle\sum_{i} a_{i} 1_{i} \cdot \sigma_{i}\right\rangle+\frac{B}{2}\left\langle\sum_{i<j} V\left(r_{i j}\right)\left(t_{i}^{z}-t_{j}^{z}\right)^{2}\left(\sigma_{i}-\sigma_{j}\right)^{2} P_{i j}^{\sigma}\right\rangle\right\}, \\
& B_{1}^{0_{V} V}(M 1) \uparrow=-\frac{3}{4 \pi}\left(\mu_{N} / 2\right)^{2}\left\{\left\langle\sum_{i} a_{i} \mathbf{l}_{i} \cdot \sigma_{i}\right\rangle+\frac{1}{2}\left\langle\sum_{i<j}\left(t_{i}^{z}-t_{j}^{z}\right)^{2}\left(W+B P_{i j}^{\sigma}\right) \sum_{\alpha=x, y, z}\left[\left(l_{i}^{\alpha}-l_{j}^{\alpha}\right),\left[\left(l_{i}^{\alpha}-l_{j}^{\alpha}\right), V\left(r_{i j}\right)\right]\right]\right\rangle\right],
\end{aligned}
$$

where the mean values are to be taken in the ground state of the Hamiltonian $H$ in Eq. (8). The first term of Eq. (10) proportional to the spin-orbit energy gives the standard Kurath's sum rule; ${ }^{5}$ the second term gives the correction to the Kurath's sum rule due to the Bartlett interaction. In the absence of spin-orbit interaction $(L-S$ coupling limit) this is the only term that remains. Likewise the LEWSR for the orbital isovector contribution contains a term proportional to the spin-orbit energy and a term that depends on the Wigner and Bartlett interactions. The total LEWSR for the isovector $M 1$ operator contains in addition a cross term coming from the interference between orbital and spin isovector operators:

$$
B_{1}(M 1) \uparrow=B_{1}^{S_{V}}(M 1) \uparrow+B_{1}^{o_{V}}(M 1) \uparrow+B_{1}^{\text {cross }}(M 1) \uparrow,
$$

with

$$
B_{1}^{\text {cross }}(M 1) \uparrow=\frac{3}{4 \pi}\left(2 \mu^{V} \mu_{N}^{2}\right)\left\{\left\langle\sum_{i} a_{i} 1_{i} \cdot \sigma_{i}\right\rangle+\frac{B}{2}\left\langle\sum_{i<j}\left(t_{i}^{z}-t_{j}^{z}\right)^{2} \sum_{\alpha} i\left(\sigma_{i} \times \sigma_{j}\right)\left[\alpha_{\alpha}\left(l_{i}^{\alpha}-l_{j}^{\alpha}, V\left(r_{i j}\right)\right]\right\rangle\right\} .\right.
$$

In the absence of spin-orbit interaction, the LEWSR is entirely given by the terms depending on the two-body interaction and only the neutron-proton part of that interaction contributes. Hence, the LEWSR is zero if there are only protons (or neutrons) in the open shells. In this case (no spin-orbit interaction) the $L-S$ coupling scheme can be used and for any pair of particles $(1,2)$ the wave function $\phi(1,2)$ can be characterized by the quantum numbers $L_{12}, S_{12}$, and $T_{12}$. In addition, with a two-body $\delta$ interaction [i.e., $V\left(r_{12}\right)=\delta\left(\mathbf{r}_{1}-\mathbf{r}_{2}\right)$ ], the spatial wave function $\phi_{L_{12}}\left(\mathbf{r}_{1}, \mathbf{r}_{2}\right)$ must be symmetric. (In this case one also has that the double commutator in Eq. (11),

$$
\sum_{\alpha}\left[\left(l_{1}^{\alpha}-l_{2}^{\alpha}\right),\left[\left(l_{1}^{\alpha}-l_{2}^{\alpha}\right), V\left(r_{12}\right)\right]\right],
$$

can be replaced by $\left.2 \delta\left(r_{1}-r_{2}\right)\left(1_{1}-1_{2}\right)^{2}\right)$. The second term in Eq. (13) will be zero. Hence, $B_{1}^{\text {cross }}$ will be zero for a spin-dependent delta interaction and no spin-orbit cou- pling. Similar sum rules can be defined for the GamowTeller operators $\mathbf{Y}_{ \pm}$.

It should be pointed out that the LEWSR's are highly model dependent and of limited value especially if the calculations are in a small space, e.g., valence nucleons in one major shell. For example, Zamick, Abbas, and Halemane ${ }^{6}$ considered $M 1$ transitions in closed $L S-j j$ shell nuclei like ${ }^{16} \mathrm{O}$ and ${ }^{40} \mathrm{Ca}$. In the absence of groundstate correlations, all $M 1$ rates and, hence, LEWSR would be zero. However, when one allows for groundstate correlations, e.g., two-particle-two-hole admixtures, especially those induced by a tensor interaction, the value of the LEWSR becomes very large. It is, in fact, much larger than say the calculated value in ${ }^{28} \mathrm{Si}$, where the 12 valence nucleons are restricted to be in the $2 s-1 d$ shell.

For spin- and isospin-independent interactions, the Wigner SU(4) group is a good symmetry group of the system and the isovector spin operators $\mathbf{Y}_{\tau}$ [see Eqs. (6) and 
(7)] connect only states belonging to the same (STY) Wigner multiplet. This is the case with the model Hamiltonian in Eqs. (8) and (9) when $a_{i}=B=0$. Then, with only a Wigner force, practically all spin isovector excitations are forbidden except for a few excitations. ${ }^{2}$ We shall turn back to this point in the next section.

A particular case of strong spin suppression and orbital enhancement is that found in Elliott's SU(3) limit, ${ }^{7}$ which is discussed in detail in Ref. 8. Because, in this limit, the shell-model calculations generate low-lying spectra of rotational character, one is tempted to connect properties concerning $M 1$ strengths, etc., that follow from shellmodel calculations with those from deformed mean-field approximations. In other words, one may relate the quenching or enhancement of orbital $M 1$ transitions in shell-model calculations to the spatial symmetries of the mean field in the corresponding Hartree-Fock (HF) calculations. The extreme case of a closed $L S$ shell illustrates this. For a closed shell there are no $M 1$ transitions. This corresponds to the fact that the mean field is spherically symmetric and commutes with the $M 1$ operator. For an open shell (considering for the moment spinand isospin-independent forces) the $M 1$ strength grows with the number of particles (or holes) in the shell up to midshell. This can be related to increasing spatial anisotropies of the corresponding mean field.

As an example of this, we recall here the results ${ }^{9}$ of shell-model calculations in the even-even $T=0 s-d$ shell nuclei $\left({ }^{20} \mathrm{Ne},{ }^{24} \mathrm{Mg},{ }^{28} \mathrm{Si},{ }^{32} \mathrm{~S}\right.$, and $\left.{ }^{36} \mathrm{Ar}\right)$ with a two-body spin-dependent delta interaction. In Ref. 9 the LEWSR for isovector $M 1$ transitions was calculated for different choices of spin-orbit splittings $a_{i}$ and of the parameter $x=B / W$. The results can be summarized as follows:

(i) For $x=a_{i}=0$ [SU(4) limit] the $B_{1}(M 1) \uparrow$ is purely orbital and grows in going from ${ }^{20} \mathrm{Ne}$ to ${ }^{24} \mathrm{Mg}$ and ${ }^{28} \mathrm{Si}$, or from ${ }^{36} \mathrm{Ar}$ to ${ }^{28} \mathrm{Si}$, in agreement with our previous remarks.

(ii) For $a_{i}=0, x=\frac{1}{3}$, one still finds the same trend of $B_{1}(M 1) \uparrow$ growing with number of particles (or holes) up to midshell, but now the spin contribution is of the same order as the orbital contribution. One also finds that

$$
B_{1}(M 1) \uparrow=B_{1}^{S_{V}}+B_{1}^{0_{V}},
$$

because as said above [see Eq. (12)] $B_{1}^{\text {cross }}$ is zero for a two-body $\delta$ interaction in the absence of spin-orbit interaction.

(iii) For $a_{i} \neq 0\left(x=0\right.$ or $\left.x=\frac{1}{3}\right)$ the spin contribution dominates over the orbital and cross contributions. The last two contributions are of the same order and of opposite sign [see Eqs. (11) and (13)]; they almost cancel each other.

\section{SELECTION RULES FROM SHELL-MODEL CALCULATIONS IN $L-S$ COUPLING LIMIT}

We performed shell-model calculations of GamowTeller and $M 1$ transitions for $A=22$ isobars $\left({ }^{22} \mathrm{O},{ }^{22} \mathrm{~F}\right.$, ${ }^{22} \mathrm{Ne}$, and ${ }^{22} \mathrm{Na}$ ), i.e., six particles in the $s-d$ shell, using zero single-particle energy splittings and different interactions. The selection rules found for $M 1$ and GamowTeller (GT) strengths are summarized in Table $I$ for the three types of forces used:

$$
\begin{aligned}
& \text { case } 1 V_{12}=-G \delta\left(\mathbf{r}_{1}-\mathbf{r}_{2}\right) ; \\
& \text { case } 2 V_{12}=-G\left(1+\frac{1}{3} P_{12}^{\sigma}\right) \delta\left(\mathbf{r}_{1}-\mathbf{r}_{2}\right) ; \\
& \text { case } 3 V_{12}=-\chi Q^{2}(1) \cdot Q^{2}(2) .
\end{aligned}
$$

The selection rules for case 1 are also shown schematically in Figs. 1 and 2 for GT and $M 1$ transitions, respectively.

Figure 1 shows that all GT strengths starting from the ground state of ${ }^{22} \mathrm{Ne}$ are zero except for the transition

$$
{ }^{22} \mathrm{Ne}\left(0^{+} 1\right) \stackrel{\mathrm{Y}_{-}}{\rightarrow}{ }^{22} \mathrm{Na}\left(1^{+} 0\right) .
$$

This transition is marked by a solid arrow in Fig. 1. It is a strong transition that exhausts the " $3(N-Z)$ " sum rule. As discussed in the previous section, the Wigner supermultiplet theory is valid in this case and hence the only allowed GT transition is the one between states belonging to the SU(4) multiplet (100). Obviously these results are independent of the type of Wigner force considered, and

TABLE I. The vanishing $0^{+}$to $1^{+}$transitions in the $A=22$ system.

Case 1. Spin-independent delta interaction $(x=0)$, degenerate s.p. energies.

(a) $B(M 1)$ are zero for $T_{i}=1\left(T_{z}=0\right)$ and $T_{f}=1$.

(b) $B(M 1)$ are purely orbital for $T_{i}=1\left(T_{z}= \pm 1\right)$ and $T_{f}=1$.

(c) $B\left(\mathrm{GT}+\right.$ ) and $B\left(\mathrm{GT}-\right.$ ) are zero for $T_{i}=1$ (any $T_{z}$ ) and $T_{f}=1$.

(d) $B(M 1)$ are purely orbital for $T_{i}=1$ (any $T_{z}$ ) and $T_{f}=2$.

(e) $B\left(\mathrm{GT}+\right.$ ) and $B\left(\mathrm{GT}-\right.$ ) are zero for $T_{i}=1$ (any $T_{z}$ ) and $T_{f}=2$.

(f) $B(M 1)$ are purely orbital for $T_{i}=2$ (any $T_{z}$ ) and $T_{f}=3$.

(g) $B\left(\mathrm{GT}+\right.$ ) and $B\left(\mathrm{GT}-\right.$ ) are zero for $T_{i}=2$ (any $T_{z}$ ) and $T_{f}=3$.

(h) $B(M 1), B\left(\mathrm{GT}+\right.$ ), and $B\left(\mathrm{GT}-\right.$ ) are zero for $T_{i}=3$ (any $T_{z}$ ) and $T_{f}=3$.

Case 2. Spin-dependent delta interaction $\left(x=\frac{1}{3}\right)$, degenerate s.p. energies.

(a) $B(M 1)$ are zero for $T_{i}=1 \quad\left(T_{z}=0\right)$ and $T_{f}=1$.

(b) $B(\mathrm{GT}+)$ and $B(\mathrm{GT}-)$ are small $\left(<10^{-3}\right)$ for $T_{i}=1$ (any $\left.T_{z}\right)$ and $T_{f}=1$.

(c) $B(M 1), B\left(\mathrm{GT}+\right.$ ), and $B\left(\mathrm{GT}-\right.$ ) are zero for $T_{i}=3$ (any $\left.T_{z}\right)$ and $T_{f}=3$.

Case 3. Quadrupole-quadrupole interaction, degenerate s.p. energies.

(same as in case 1) 


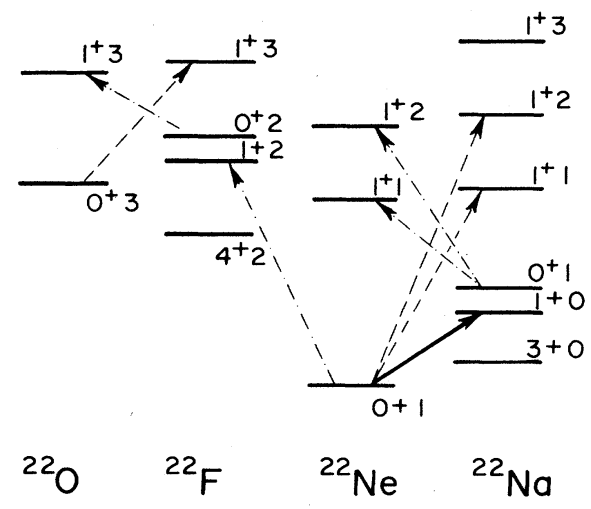

FIG. 1. The vanishing $B(\mathrm{GT}+)$ (dash-dotted) and $B(\mathrm{GT}-$ ) (dashed) transitions of the $A=22$ system (with $T_{z} \geq 0$ ) in the $s-d$ shell-model calculation when the spin-independent delta interaction is used and the single-particle energies are set to zero. The pictures for $T_{z} \leq 0$ and $T_{z} \geq 0$ are symmetric about $T_{z}=0$. The solid line represents the only nonvanishing GT transition from the ground state of ${ }^{22} \mathrm{Ne}$. It exhausts the $3(N-Z)$ sum rule. Other possible transitions not connected by lines in the picture are allowed.

therefore the selection rules for Gamow-Teller transitions are exactly the same in cases 1 and 3 . When the spindependent $\delta$ interaction is included (case 2 ) the multiplets $S T Y$ are destroyed in the sense that $Y$ is no longer a good quantum number ${ }^{2}$ and most GT transitions are allowed (see Table I). However, in this case $L, S$, and $T$ are still good quantum numbers, and therefore, one still has the selection rule $B(\mathrm{GT} \pm)=0$ for $T_{i}=T_{f}=3$. To understand why this is so, we have to keep in mind that for the six-particle system the states with $T=T_{\max }=3, T_{z}=3$, two are completely symmetric in isospin space and, because of the $\delta$ force, are spatially symmetric. Hence, they are all $S=0$ states and are not connected by the $\mathbf{Y}_{ \pm}$ operators.

$$
\left\langle I^{\prime} T^{\prime} T_{z}\left\|\mu_{V}\right\| I T T_{z}\right\rangle=(-1)^{T^{\prime}-T_{z}}\left(\begin{array}{ccc}
T^{\prime} & 1 & T \\
-T_{z} & 0 & T_{z}
\end{array}\right]\left\langle I^{\prime} T^{\prime}\left|\left\|\mu_{V}\right\|\right| I T\right\rangle
$$

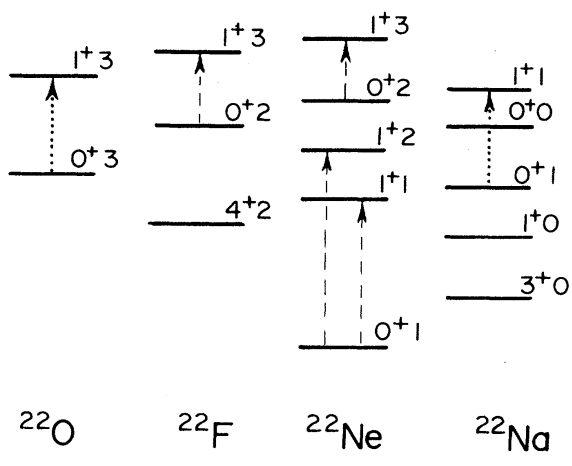

FIG. 2. Same as Fig. 1 but for the vanishing (dotted) and the purely orbital (dashed) $B(M 1)$ transitions.

A similar argument explains why $B(M 1) \uparrow=0$ in all three cases for ${ }^{22} \mathrm{O}$ (see Table $\mathrm{I}$ and Fig. 2). The $0^{+} 3 \stackrel{M 1}{\rightarrow} 1^{+} 3$ excitation could only be orbital, but since neutrons have no charge, $B(M 1) \uparrow=0$. It is also worth recalling here that for identical particles (only neutrons in the ${ }^{22} \mathrm{O}$ case) the isovector and isoscalar excitations are the same, and, as discussed in the previous section, the isovector LEWSR is easily shown to be zero.

Figure 2 also shows that in case 1 many (but not all) allowed $M 1$ transitions are purely orbital. As in the case of GT transition, the lack of spin strength here is connected to SU(4) symmetry and does not prevail when the spindependent interaction is taken into account. However, the selection rule (a) $[B(M 1) \uparrow=0$ for $T_{i}=T_{f}=1, T_{z}=0$ ] prevails in all three cases (see Table I and Fig. 2). In Fig. 2 one sees that the transition $0^{+} 1 \stackrel{M 1}{\rightarrow} 1^{+} 1$ in ${ }^{22} \mathrm{Ne}$ is purely orbital, but the corresponding transition in ${ }^{22} \mathrm{Na}$ is zero. This selection rule comes from isospin SU(2) algebra and is easily understood if one takes reduced matrix elements of Eq. (1) in isospin space:
The three- $j$ symbol in Eq. (14) is zero for $T^{\prime}=T$ and $T_{z}=0$. In particular,

$$
(-1)^{T^{\prime}-T_{z}}\left(\begin{array}{ccc}
1 & 1 & 1 \\
-T_{z} & 0 & T_{z}
\end{array}\right)=T_{z} / \sqrt{6}
$$

is zero for ${ }^{22} \mathrm{Na}$ and $1 / \sqrt{6}$ for ${ }^{22} \mathrm{Ne}$. Hence, the selection rule (a) is a particular case of the general selection rule that says that there are no $\Delta T=0$, isovector $M 1$ transitions in self-conjugate nuclei. ${ }^{10}$
The same coefficient in Eq. (15) enters in GamowTeller transitions between ${ }^{22} \mathrm{Ne}$ and ${ }^{22} \mathrm{Na}$ isobars for $T_{i}=T_{f}=1$. As said before, in cases 1 and 3 these transitions are forbidden because of SU(4) symmetry, but even in case 2 these transitions are very small because the $0^{+} 1$ and $1^{+} 1$ states are still predominantly $S=0$ states. It is also interesting to note that the $M 1$ transitions between $T_{i}=2$ and $T_{f}=3$ states in ${ }^{22} \mathrm{Ne}$ and ${ }^{22} \mathrm{~F}$, which in cases 1 and 3 are purely orbital, are much smaller (by an order of $10^{-3}$ ) in case 3 (quadrupole-quadrupole interaction) than that in case 1 ( $\delta$ interaction). 


\section{NUCLEAR DEFORMATION-ONE-BODY APPROACH}

In the previous section we considered spin and orbital selection rules in terms of two-body interactions. In this section we will focus on the one-body aspects by considering the deformed mean field. We will consider how the nuclear shape affects the spin properties in a nucleus. To this end we compute total orbital and spin strengths for the case of deformed harmonic oscillator mean fields. We consider first the case of axial symmetry $\left(\omega_{x}=\omega_{y}>\omega_{z}\right.$, prolate) and then the triaxial case $\left(\omega_{x}>\omega_{y}>\omega_{z}\right)$. As in the previous section, calculations are presented for eveneven nuclei with the $N=2$ shell open. The problem of orbital enhancement has been previously considered in the context of projected Hartree-Fock by Moya de Guerra et al. ${ }^{11}$

\section{A. Axial symmetry}

To stress analogy with the $L-S$ coupling limit considered in the previous section, we consider Nilsson model wave functions in the asymptotic limit. In this limit the single-particle levels are characterized by $\Omega^{\pi}\left[N n_{z} \Lambda\right]$ or, equivalently,

$$
\Omega^{\pi}\left[n_{r} n_{z} \Lambda\right]\left(\Omega=\Lambda+\Sigma \geq \frac{1}{2}, N=2 n_{r}+n_{z}+\Lambda\right) .
$$

The order of the twofold degenerate levels in a given $N$ shell is $\frac{1}{2}[N N 0], \frac{3}{2}[N N-11], \frac{1}{2}[N N-11], \frac{5}{2}[N N-22]$, $\frac{3}{2}[N N-22], \frac{1}{2}[N N-20], \ldots,$. In this basis $l_{z}$ and $s_{z}$ are diagonal and the total $M 1$ strength is

$B(M 1) \uparrow=\frac{3}{4 \pi}\left(\left\langle\phi_{0}\left|\mu_{-} \mu_{+}\right| \phi_{0}\right\rangle-\left\langle\phi_{S}\left|\mu_{+}\right| \phi_{0}\right\rangle^{2}\right)$,
TABLE II. Selection rules and matrix elements of $l_{+}$in the cylindrical basis $\left(\beta_{z}=\sqrt{\left(M \omega_{z} / \hbar\right)}=1 / b_{z}, \quad \beta_{\perp}=\sqrt{\left(M \omega_{1} / \hbar\right)}\right.$ $=1 / b_{1}$, and $\beta^{ \pm}=\beta_{\perp} / \beta_{z} \pm \beta_{z} / \beta_{1}$.

\begin{tabular}{llll}
\hline$N^{\prime}$ & $n_{r}^{\prime}$ & $n_{z}^{\prime}$ & $\left\langle n_{r}^{\prime} n_{z}^{\prime} \Lambda+1 \Omega+1\left|l_{+}\right| n_{r} n_{z} \Lambda \Omega\right\rangle$
\end{tabular}

\begin{tabular}{llcc}
$N$ & $n_{r}$ & $n_{z}-1$ & $-\beta^{+} \sqrt{\left(n_{r}+\Lambda+1\right) n_{z} / 2}$ \\
$N$ & $n_{r}-1$ & $n_{z}+1$ & $-\beta^{+} \sqrt{n_{r}\left(n_{z}+1\right) / 2}$ \\
$N+2$ & $n_{r}$ & $n_{z}+1$ & $-\beta^{-} \sqrt{\left(n_{r}+\Lambda+1\right)\left(n_{z}+1\right) / 2}$ \\
$N-2$ & $n_{r}-1$ & $n_{z}-1$ & $-\beta^{-} \sqrt{n_{r} n_{z} / 2}$ \\
\hline
\end{tabular}

where the second term represents the correction due to the spurious state

$$
\phi_{S}=\frac{1}{N} J_{+}\left|\phi_{0}\right\rangle, \quad N^{2}=\left\langle\phi_{0}\left|J^{2}\right| \phi_{0}\right\rangle,
$$

and $\phi_{0}$ is the intrinsic ground state.

Using the selection rules in Table II for the $l_{+}$operator, as well as

$$
s_{ \pm}\left|n_{r} n_{z} \Lambda \Sigma\right\rangle=\left|n_{r} n_{z} \Lambda \Sigma \pm \frac{1}{2}\right\rangle \delta_{\Lambda, \Omega \pm 1 / 2}
$$

the matrix elements in Eq. (16) can be easily computed for any particular ground-state configuration $\phi_{0}$. We assume a closed core and $2 n_{\pi}$ protons and $2 n_{v}$ neutrons in the open shell $N$. We denote by $h_{\rho}(\rho=\pi, v)$ the occupied states in the $N$ shell and by $p_{\rho}$ the empty levels in the $N$ and $N+2$ shells (see Table II) and write

$$
\left\langle\phi_{0}\left|\mu_{-} \mu_{+}\right| \phi_{0}\right\rangle=\sum_{\rho=\pi, v} \sum_{\rho_{\rho} h_{\rho}}\left(\left|\left\langle p_{\rho}\left|\mu_{+}\right| h_{\rho}\right\rangle\right|^{2}+\left|\left\langle p_{\rho}\left|\mu_{-}\right| h_{\rho}\right\rangle\right|^{2}+\left|\left\langle p_{\rho}\left|\mu_{+}\right| \bar{h}_{\rho}\right\rangle\right|^{2}\right) .
$$

The states $h_{\rho}, p_{\rho}$ in Eq. (18) are defined so that their $\Omega$ value is greater than zero ( $\Omega_{\rho} \geq \frac{1}{2}$ ) and $\bar{h}_{\rho}$ is the time reverse of $h_{\rho}$. A similar expression holds for the spurious correction

$$
\left\langle\phi_{S}\left|\mu_{+}\right| \phi_{0}\right\rangle=\frac{1}{N} \sum_{\rho} \sum_{p_{\rho} h_{\rho}}\left(\left\langle p_{\rho}\left|j_{+}\right| h_{\rho}\right\rangle\left\langle p_{\rho}\left|\mu_{+}\right| h_{\rho}\right\rangle+\left\langle p_{\rho}\left|j_{-}\right| h_{\rho}\right\rangle\left\langle p_{\rho}\left|\mu_{-}\right| h_{\rho}\right\rangle+\left\langle p_{\rho}\left|j_{+}\right| \bar{h}_{\rho}\right\rangle\left\langle p_{\rho}\left|\mu_{+}\right| \bar{h}_{\rho}\right\rangle\right)
$$

and normalization factor

$$
\begin{gathered}
N^{2}=\sum_{\rho} \sum_{p_{\rho} h_{\rho}}\left(\left|\left\langle p_{\rho}\left|j_{+}\right| h_{\rho}\right\rangle\right|^{2}+\left|\left\langle p_{\rho}\left|j_{-}\right| h_{\rho}\right\rangle\right|^{2}\right. \\
\left.+\left|\left\langle p_{\rho}\left|j_{+}\right| \bar{h}_{\rho}\right\rangle\right|^{2}\right) .
\end{gathered}
$$

From these equations $[(16)-(21)]$ it is easy to see that if there are only two neutrons and/or protons in the open shell $N$, the total spin strength will be zero. Indeed, in that case there is only one $h_{\rho}$ state entering in Eqs. (19)-(21), the one corresponding to the lowest level $\frac{1}{2}$ [NNO] which cannot be connected by the $S_{ \pm}$operators to any empty state $p_{\rho}$.

Hence, axially symmetric nuclear deformation can be understood as a mechanism for spin suppression in some particular cases, like the one just considered of only two neutrons and/or only two protons outside closed shells. If more nucleons are added in the open shell $N$, other states $\left(\frac{3}{2}[N N-11], \ldots,\right)$ are occupied that may be connected by the $S_{ \pm}$operators to empty states. In those cases the spin strength will remain finite at any deformation. 
As an example, consider the cases of ${ }^{20} \mathrm{Ne}$ and ${ }^{22} \mathrm{Ne}$. For ${ }^{20} \mathrm{Ne}$ we have two protons and two neutrons in the $\frac{1}{2}[220]$ level and it is easy to see from Table II that the nonzero matrix elements entering in Eqs. (19) - (21) are

$$
\begin{aligned}
& \left\langle 211 \frac{3}{2}\left|l_{+}\right| 220 \frac{1}{2}\right\rangle=\left\langle 211 \frac{1}{2}\left|l_{+}\right| \overline{220 \frac{1}{2}}\right\rangle=-\beta_{+}, \\
& \left\langle 431 \frac{3}{2}\left|l_{+}\right| 220 \frac{1}{2}\right\rangle=\left\langle 431 \frac{1}{2}\left|l_{+}\right| \overline{220 \frac{1}{2}}\right\rangle=-\sqrt{3 / 2} \beta_{-},
\end{aligned}
$$

for both proton and neutron states. After substitution of Eq. (22) into Eqs. (19)-(21) we find, taking bare $g_{l}$ values,

$$
\begin{aligned}
\left\langle\phi_{0}\left|\mu_{-} \mu_{+}\right| \phi_{0}\right\rangle & =\left\langle\phi_{0}\left|L_{-} L_{+}\right| \phi_{0}\right\rangle_{\pi} \\
& =2\left|\left\langle\phi_{S}\left|\mu_{+}\right| \phi_{0}\right\rangle\right|^{2}=\left(2 \beta_{+}^{2}+3 \beta_{-}^{2}\right) \mu_{N}^{2},
\end{aligned}
$$

which gives a purely orbital $M 1$ strength,

$$
[B(M 1) \uparrow]_{\text {total }}^{20}=\frac{3}{4 \pi}\left(\beta_{+}^{2}+\frac{3}{2} \beta_{-}^{2}\right) \mu_{N}^{2}
$$

From this expression one also sees that this purely orbital strength tends to increase with deformation. For small deformation $\beta_{-}^{2} \approx 0$ and $\beta_{+}^{2} \approx 4$, but for large deformation $\beta_{+}^{2}$ and $\beta_{-}^{2}$ increase as $\omega_{\perp} / \omega_{z}$. For instance, for superdeformed ${ }^{20} \mathrm{Ne}\left(\omega_{1} / \omega_{z}=2\right)$ one has $\beta_{+}^{2}+\frac{3}{2} \beta_{-}^{2}=5.75$.

For ${ }^{22} \mathrm{Ne}$ we have, in addition, two neutrons in the $\frac{3}{2}$ [211] level. For protons, the nonzero matrix elements are again those in Eq. (22), but for neutrons the nonzero matrix elements are now

$$
\begin{aligned}
& \left\langle 431 \frac{3}{2}\left|l_{+}\right| 220 \frac{1}{2}\right\rangle=\left\langle 431 \frac{1}{2} \mid l_{+} \overline{220 \frac{1}{2}}\right\rangle=-\sqrt{3 / 2} \beta_{-} ; \\
& \left\langle 211 \frac{1}{2}\left|l_{+}\right| \overline{220 \frac{1}{2}}\right\rangle=\left\langle 202 \frac{5}{2}\left|l_{+}\right| 211 \frac{3}{2}\right\rangle=-\beta_{+} ; \\
& \left\langle 422 \frac{5}{2}\left|l_{+}\right| 211 \frac{3}{2}\right\rangle=-\sqrt{2} \beta_{-} ; \\
& \left\langle 420 \frac{1}{2}\left|l_{-}\right| 211 \frac{3}{2}\right\rangle=-\beta_{-} ; \\
& \left\langle 200 \frac{1}{2}\left|l_{-}\right| 211 \frac{3}{2}\right\rangle=-\beta_{+} / \sqrt{2} ; \\
& \left\langle 211 \frac{1}{2}\left|s_{-}\right| 211 \frac{3}{2}\right\rangle=1
\end{aligned}
$$

where, as in Eq. (22), we write occupied states to the right and empty states to the left. Clearly, there is, in this case, a spin contribution to the total strength, the latter is given by

$$
\begin{aligned}
\left.B(M 1) \uparrow\right|_{\text {total }} ^{22} \mathrm{Ne}=\frac{3 \mu_{N}^{2}}{4 \pi} & {\left[2 \beta_{+}^{2}+3 \beta_{-}^{2}+\left(g_{s}^{v}\right)^{2}\right.} \\
& \left.-\left(2 \beta_{+}^{2}+3 \beta_{-}^{2}+g_{s}^{v}\right)^{2} / N^{2}\right],
\end{aligned}
$$

where we used again bare $g_{l}$ values, and the normalization factor of the spurious correction is now

$$
N^{2}=\frac{9}{2} \beta_{+}^{2}+9 \beta_{-}^{2}+1 \text {. }
$$

Hence, we see that, in this case, the $M 1$ orbital contribution still grows with deformation as $\omega_{1} / \omega_{z}$, but for any deformation there is always a finite spin contribution. For instance, for $\omega_{1} / \omega_{z}=2$, one has an orbital contribution of

$$
6.3 \times \frac{3}{4 \pi} \mu_{N}^{2}
$$

which is less than half the total $B(M 1) \uparrow$ for the same deformation when the spin contribution is included.

It should be mentioned that the orbital enhancement for $M 1$ transitions does not extend to magnetic moments of $K \neq \frac{1}{2}$ bands. For the orbital part of magnetic moments we evaluate, in the intrinsic state, the expectation value of $L_{z}$. In more detail the matrix element is

$$
-i \hbar\left\langle\psi\left(\beta_{\perp} x, \beta_{\perp} y, \beta_{z} z\right)[x(\partial / \partial y)-y(\partial / \partial x)] \psi\left(\beta_{\perp} x, \beta_{\perp} y, \beta_{z} z\right)\right\rangle .
$$

Introducing $\hat{x}=\beta_{\perp} x$, etc., this becomes

$$
-i \hbar\langle\psi(\hat{x}, \hat{y}, \hat{z})[\hat{x}(\partial / \partial \hat{y})-\hat{y}(\partial / \partial \hat{x})] \psi(\hat{x}, \hat{y}, \hat{z})\rangle .
$$

Clearly, this expression does not depend on $\beta_{1}$ or $\beta_{z}$.

This difference in the behavior of magnetic moments and magnetic dipole transitions may help to distinguish this deformation mechanism from another source of orbital renormalization-meson exchange currents. Although the calculations of the latter effects are complex the effects can be approximated by replacing the orbital term $g_{l} l$ by $\left(g_{l}+\delta g_{l}\right) l+g_{p}\left[Y^{2} s\right]^{\lambda=1}$, where $\delta g_{l}$ is approximately equal to $0.2 \mathrm{~N} / A$ for a proton and $-0.2 Z / A$ for a neutron. This renormalization affects both magnetic moments and $M 1$ transitions. In order to disentangle the relative contributions of the deformation mechanism and exchange current mechanism one will have to carefully analyze both the magnetic dipole moments and the magnetic dipole transitions which have a strong orbital content.

\section{B. Triaxial case}

In this case we label the single-particle levels by $\left(n_{x}, n_{y}, n_{z}\right)$ with degenerate single-particle states for $\Sigma=+\frac{1}{2}$ and $\Sigma=-\frac{1}{2}$. For a given $N$ shell, the sequence of levels is now $(00 N),(01 N-1),(10 N-1),(02 N-2)$, $(11 N-2),(20 N-2), \ldots$, and $(N 00)$. The selection rules and matrix elements for $l_{\mu}$ operators are shown in Table III. Considering again a given state configuration with $2 n_{\pi}$ protons and $2 n_{v}$ neutrons in such a shell, an analysis similar to the one in the previous section shows 
TABLE III. Selection rules and matrix elements of $l_{i}\left(\beta_{i j}^{ \pm}=\beta_{i} / \beta_{j} \pm \beta_{j} / \beta_{i}\right)$.

\begin{tabular}{|c|c|c|c|c|}
\hline$N^{\prime}$ & $n_{x}^{\prime}$ & $n_{y}^{\prime}$ & $n_{z}^{\prime}$ & $\left\langle n_{x}^{\prime} n_{y}^{\prime} n_{z}^{\prime}\left|2 i l_{x}\right| n_{x} n_{y} n_{z}\right\rangle$ \\
\hline$N$ & $n_{x}$ & $n_{y}+1$ & $n_{z}-1$ & $\sqrt{n_{z}\left(n_{y}+1\right)} \beta_{y z}^{+}$ \\
\hline$N$ & $n_{x}$ & $n_{y}-1$ & $n_{z}+1$ & $\sqrt{n_{y}\left(n_{z}+1\right)}\left(-\beta_{y z}^{+}\right)$ \\
\hline$N+2$ & $n_{x}$ & $n_{y}+1$ & $n_{z}+1$ & $\sqrt{\left(n_{z}+1\right)\left(n_{y}+1\right)} \beta_{y z}^{-}$ \\
\hline \multirow[t]{2}{*}{$N-2$} & $n_{x}$ & $n_{y}-1$ & $n_{z}-1$ & $\sqrt{n_{y} n_{z}}\left(-\beta_{y z}^{-}\right)$ \\
\hline & & & & $\left\langle n_{x}^{\prime} n_{y}^{\prime} n_{z}^{\prime}\left|2 i l_{y}\right| n_{x} n_{y} n_{z}\right\rangle$ \\
\hline$N$ & $n_{x}+1$ & $n_{y}$ & $n_{z}-1$ & $\sqrt{n_{z}\left(n_{x}+1\right)}\left(-\beta_{x z}^{+}\right)$ \\
\hline$N$ & $n_{x}-1$ & $n_{y}$ & $n_{z}+1$ & $\sqrt{n_{x}\left(n_{z}+1\right)} \beta_{x z}^{+}$ \\
\hline$N+2$ & $n_{x}+1$ & $n_{y}$ & $n_{z}+1$ & $\sqrt{\left(n_{x}+1\right)\left(n_{z}+1\right)}\left(-\beta_{x z}^{-}\right)$ \\
\hline \multirow[t]{2}{*}{$N-2$} & $n_{x}-1$ & $n_{y}$ & $n_{z}-1$ & $\sqrt{n_{x} n_{z}} \beta_{x z}^{-}$ \\
\hline & & & & $\left\langle n_{x}^{\prime} n_{y}^{\prime} n_{z}^{\prime}\left|2 i l_{z}\right| n_{x} n_{y} n_{z}\right\rangle$ \\
\hline$N$ & $n_{x}+1$ & $n_{y}-1$ & $n_{z}$ & $\sqrt{n_{y}\left(n_{x}+1\right)} \beta_{x y}^{+}$ \\
\hline$N$ & $n_{x}-1$ & $n_{y}+1$ & $n_{z}$ & $\sqrt{n_{x}\left(n_{y}+1\right)}\left(-\beta_{x y}^{+}\right)$ \\
\hline$N+2$ & $n_{x}+1$ & $n_{y}+1$ & $n_{z}$ & $\sqrt{\left(n_{x}+1\right)\left(n_{y}+1\right)} \beta_{x y}^{-}$ \\
\hline$N-2$ & $n_{x}-1$ & $n_{y}-1$ & $n_{z}$ & $\sqrt{n_{x} n_{y}}\left(-\beta_{x y}^{-}\right)$ \\
\hline
\end{tabular}

that in this case the orbital $M 1$ strength has contributions proportional to

$$
\left(\beta_{i j}^{+}\right)^{2}(i \neq j, i, j=x, y, z)
$$

from particle-hole excitations within the same major shell $N$, and contributions proportional to $\left(\beta_{i j}^{-}\right)^{2}$ from excitations to the $N+2$ shell (see Table III). Therefore the orbital $M 1$ strength grows again with deformation. On the other hand, for the spin operator $s_{\mu}$ the matrix elements are diagonal in $n_{x}, n_{y}$, and $n_{z}$ :

$$
\left\langle n_{x}^{\prime} n_{y}^{\prime} n_{z}^{\prime} \Sigma^{\prime}\left|s_{\mu}\right| n_{x} n_{y} n_{z} \Sigma\right\rangle=\delta_{n_{x}^{\prime} n_{x}} \delta_{n_{y}^{\prime} n_{y}} \delta_{n_{z}^{\prime} n_{z}}\left\langle\Sigma^{\prime}\left|s_{\mu}\right| \Sigma\right\rangle \text {. }
$$

Therefore there are no particle-hole excitations due to the spin operator and the total $B(M 1) \uparrow$ strength is purely orbital.

In the restricted $N=2$ space (i.e., neglecting contributions proportional to $\beta_{i j}^{-}$) the total $M 1$ strength for ${ }^{22} \mathrm{Ne}$ in the triaxial case is

$$
B(M 1) \uparrow=\frac{3}{4 \pi} \mu_{N}^{2}\left[\left(\beta_{y z}^{+}\right)^{2}+\left(\beta_{x z}^{+}\right)^{2}\right]\left[1-\frac{\left(\beta_{y z}^{+}\right)^{2}+\left(\beta_{x z}^{+}\right)^{2}}{2\left(\beta_{y z}^{+}\right)^{2}+\frac{5}{2}\left(\beta_{x z}^{+}\right)^{2}+\frac{1}{2}\left(\beta_{x y}^{+}\right)^{2}}\right] .
$$

In Eq. (27) (triaxial asymptotic) there is no spin contribution to $B(M 1) \uparrow$. This is in striking contrast to Eq. (25) (axial asymptotic) where the spin contribution is substantial.

We can make a connection with the two-body approach in Sec. II by noting that a Hartree-Fock calculation with a pure Wigner interaction will necessarily lead to a triaxial solution for ${ }^{22} \mathrm{Ne}$ (or ${ }^{24} \mathrm{Mg}$ ). Thus, things hang together nicely. In both the shell-model calculation and the one-body approach, the spin contributions to $B(M 1)$ transitions from the ground state vanish and the transitions are purely orbital.

Summarizing this part we may then say that while axially symmetric deformation is a mechanism for spin 

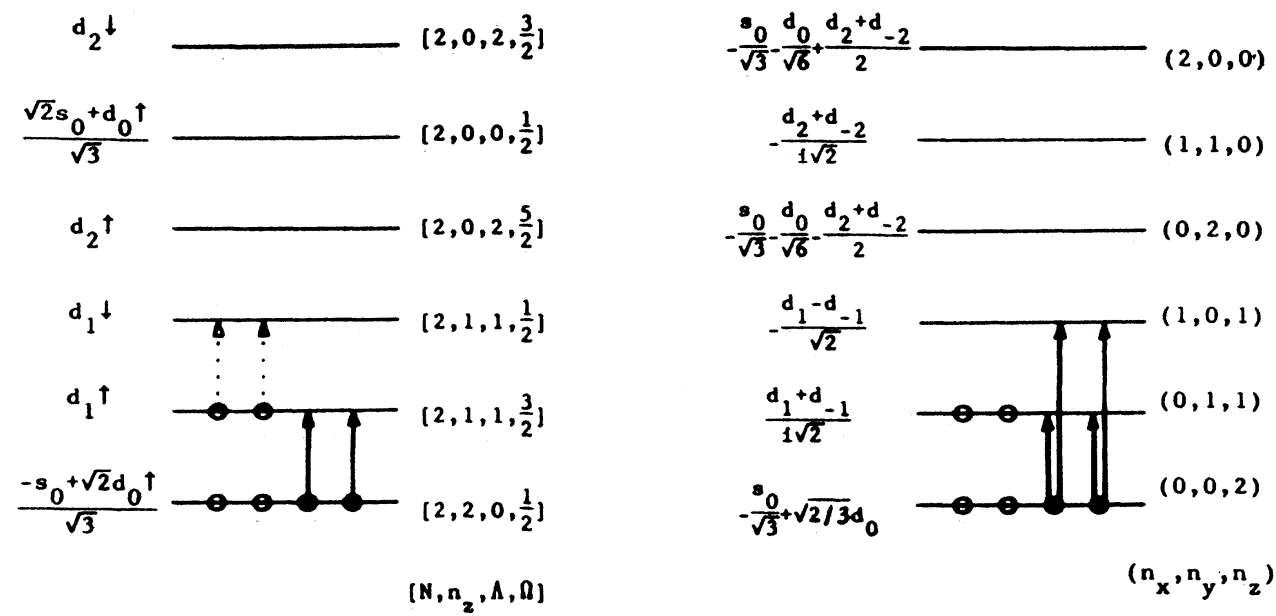

FIG. 3. Schematic representation of possible $M 1$ excitations in ${ }^{22} \mathrm{Ne}$ for axial and triaxial shapes within the major shell $N=2$. Occupied orbits are marked by solid circles for protons and by open circles for neutrons. Orbital and spin excitations are shown by solid and dotted lines, respectively. The representation of the orbital in the restricted $N=2 s-d$ basis is indicated at the left of each level.

suppression in some particular cases like ${ }^{20} \mathrm{Ne}$, triaxial deformation provides a more stringent mechanism that causes complete suppression of the spin strength in the asymptotic limit. This difference between axial and triaxial deformations is illustrated schematically in Fig. 3 for ${ }^{22} \mathrm{Ne}$, where for simplicity we consider only transitions within the restricted $N=2$ space.

\section{What is the correct picture - axial or triaxial?}

We have seen that there is a large difference in the spin contribution to $B(M 1)$ depending on whether a nucleus is axially or triaxially deformed. We will of course have to treat every nucleus on an individual basis and keep open both possibilities.

For the specific case of ${ }^{22} \mathrm{Ne}$, early Hartree-Fock results tended to yield triaxial solutions. However, with more modern Hartree-Fock calculations, in which sufficiently strong spin-orbit interactions are included, the lowest energy solution has axial symmetry. ${ }^{12,13}$

Independent, albeit circumstantial, evidence for axial symmetry comes from the comparison, performed by Liu et al., ${ }^{14}$ of the $B(M 1)$ strength, both spin and orbital, in an axial Nilsson model calculation and in the shell model. The shell-model results with the OXBASH computer code $^{15}$ have generally fit a wide variety of data and there- fore should be reliable. Both calculations, shell-model and Nilsson, predict substantially larger spin contributions to $B(M 1) \uparrow$ in ${ }^{22} \mathrm{Ne}$ than in ${ }^{20} \mathrm{Ne}$. The relative magnitudes of spin and orbital contributions in the two calculations are in fair agreement. This suggests that ${ }^{22} \mathrm{Ne}$ is not a triaxial nucleus and there should therefore not be a substantial reduction in the spin strength.

It is, however, possible that certain selected heavier nuclei do have triaxial shapes and that therefore the spin strengths in these nuclei will be lower than what one would expect. For example, we may ask, in the context of double beta-decay problem, whether a calculated suppression of double beta-decay matrix elements, as obtained by J. Engel et al., ${ }^{16}$ which they attribute to a neutron-proton interaction (probably in a $J=1 T=0$ state) can be equivalently explained in a one-body field picture, as due to the onset of triaxiality or gamma instability. We plan to look at this problem in the near future.

\section{ACKNOWLEDGMENTS}

This work was supported by a NATO Collaborative Research Grant (0702/87) and by the Department of Energy DE-FG05-86ER40299. One of us (E.M.G.) is also indebted to DGICYT (Spain) for partial financial support under Contract No. PB8710311.
${ }^{1}$ G. Morpurgo, Phys. Rev. 110, 72 (1958).

${ }^{2}$ E. P. Wigner, Phys. Rev. 51, 106 (1937); 56, 519 (1939).

${ }^{3}$ P. Bohle, A. Richter, W. Steffen, A. E. L. Dieperink, N. Lo Iudice, J. Palumbo, and O. Schalter, Phys. Lett. 137B, 27 (1984); D. Bohle, C. Kuchler, A. Richter, and W. Steffen, ibid. 148B, 360 (1984); U. E. P. Berg et al., ibid. 149B, 269 (1985).

${ }^{4}$ E. Lipparini and S. Stringari, Phys. Rep. (to be published). ${ }^{5}$ D. Kurath, Phys. Rev. 130, 525 (1963).

${ }^{6}$ L. Zamick, A. Abbas, and T. R. Halemane, Phys. Lett. 103B,
87 (1981).

${ }^{7}$ J. P. Elliott, Proc. R. Soc. London, Ser. A 245, 128 (1958); 245, 562 (1958).

${ }^{8}$ A. Proves, J. Retamosa, and E. Moya de Guerra, Phys. Rev. C (to be published); A. Poves and E. Moya de Guerra, in Proceedings of the Capri Conference, Capri, Italy, 1988 (unpublished).

${ }^{9}$ T. R. Halemane, A. Abbas, and L. Zamick, J. Phys. G 7, 163 (1981).

${ }^{10}$ P. J. Brussard and P. W. M. Glaudemans, Shell-Model Appli- 
cations in Nuclear Spectroscopy (North-Holland, New York, 1977).

${ }^{11}$ E. Moya de Guerra, P. Sarriguren, and J. M. Udias, Phys. Lett. B 196, 409 (1987); A. E. L. D. Dieperink and E. Moya de Guerra, ibid. 189, 267 (1987).

${ }_{12}$ P. Bonche, H. Flocard, and P. H. Heenen, Nucl. Phys. A467, 115 (1987).
${ }^{13}$ W. Koepf and P. Ring, Phys. Lett. B 212, 397 (1988).

${ }^{14}$ H. Liu and L. Zamick, Phys. Rev. C 36, 2057 (1987).

${ }^{15}$ W. M. D. Rae, A. Eschegoyen, N. S. Goodwin, and B. A. Brown (unpublished).

16J. Engel, P. Vogel, and M. R. Zirnbauer, Phys. Rev. C 37, 731 (1988). 